\title{
Dielectric behavior of acetonitrile + methanol binary mixtures at microwave frequency
}

\author{
A.P. Jogdand ${ }^{1}$ and Dr. P.L. Kadam ${ }^{2}$ \\ 1 and 2. Physics research laboratory, Yeshwant Mahavidyalaya, Nanded-431605, Maharashtra, India
}

\begin{abstract}
Values of dielectric constant $\left(\in^{\prime}\right)$ and dielectric loss $\left(\in \in^{\prime}\right)$ have been experimentally determined for binary liquid mixtures of acetonitrile + methanol at $10.75 \mathrm{GHz}$ microwave frequencies at $30^{\circ} \mathrm{C}$. The values of $\in^{\prime}$ and $\in "$ have been used to evaluate the loss tangent $(\tan \delta)$, molar polarization $\left(P_{12}\right)$, apparent polarization $\left(P_{2}\right)$ and a.c. conductivity $(\sigma p)$.
\end{abstract}

From the studied system indicates that the solute-solvent type of interaction taking place.

Density, viscosity and refractive index measurement of binary liquid mixtures were carried out at $30^{\circ} \mathrm{C}$. The values of viscosity have been used to evaluate the activation energy (Ea). The surface tension has been experimentally determined for pure liquid and binary liquid mixtures at $30^{\circ} \mathrm{C}$.These parameters have been used to explain the formation of 1:1 complex in the system.

Keywords: Binary mixture, Dielectric parameters, Molar polarization, Activation energy, a.c. conductivity, Molecular interaction

\section{Introduction}

Alcohols are industrially and scientifically important organic compounds and their physical and chemical properties are largely determined by $-\mathrm{OH}$ group. Alcohols are strongly associated in solutions because of dipole-dipole interaction and hydrogen bonding ${ }^{1}$.

Methanol is used as solvent and fuel due to synthetic and industrial applications. Therefore it seemed important to examine the dielectric behavior of methanol with acetonitrile.

A dielectric investigation of solutions containing varying amounts of interacting molecules helps to detect the formation and composition of complexes in them ${ }^{2}$. A survey of the literature shows that a few workers have tried to investigate some binary systems taking nitriles as one of the constituent components in the binary mixtures. Though the information in this field is steadily being enlarged by a number of workers. ${ }^{3-7}$ The nature of complex formation in binary mixtures is still far from clear. With this in view, from experimental results dielectric constant $\left(\in \in^{\prime}\right)$, dielectric loss $(\in ")$, loss tangent $(\tan \delta)$, molar polarization $\left(\mathrm{P}_{12}\right)$, apparent polarization $\left(\mathrm{P}_{2}\right)$, activation energy $(\mathrm{Ea})$, a.c. conductivity have been calculated. In this paper we also report the range of physical properties includes density, refractive index, viscosity and surface tension.

The dielectric study of acetonitrile and methanol binary liquid mixtures using frequency domain reflectometry (FDR) have not been carried out in the past.

Hence, we felt that the present investigation which may provide useful information about the formation of complexes in the acetonitrile + methanol binary liquid mixtures at $30^{\circ} \mathrm{C}$.

\section{Experimental details}

The dissipation factor $(D)$, dielectric constant $\left(\epsilon^{\prime}\right)$ and dielectric loss $(\in ")$ were measured using Surber's technique ${ }^{8,9}$ of measuring the reflection coefficient from the air-dielectric boundary of the liquid in the microwave X-band at $10.75 \mathrm{GHz}$ frequency and at $30^{\circ} \mathrm{C}$ temperature.

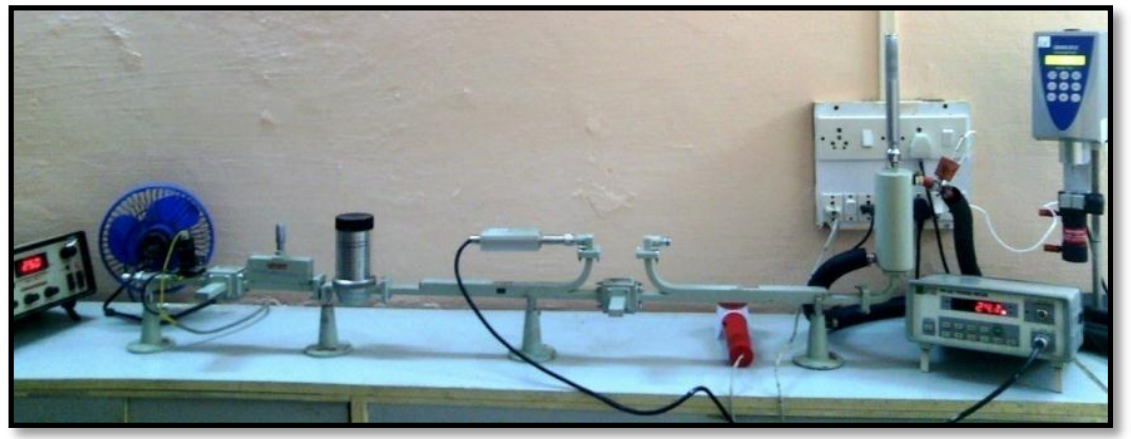

Figure 1. The experimental setup of microwave X-band bench for the measurement of $\in{ }^{\prime}$ and $\in$ " 
The experimental setup is shown in figure 1. The dielectric closed cell has a movable short. To hold the liquid in the cell, a thin mica window, whose VSWR and attenuation were neglected, was introduced between the cell and the rest of the microwave bench. Here source of reflex klystron 2 K 25 (USSR) was used

A plunger wave guide is converted into a cavity by introducing a coupling hole in the entrance and shorting the other end with the calibrated plunger. The sample occupies the entire volume of the cavity the frequency is kept constant and the length of the plunger cavity is changed. Hence, several nodes appear as one increase the length of the cavity plunger, whenever the length of the cavity equals the half integral multiples of the guide wave length inside the medium. The plunger wave guide resonates the distance through which the plunger is moved between the successive cavity nodes gives half of the wave length $(\lambda d)$ of the microwave inside the medium.

The measurement of reflected power at resonance gives the attenuation coefficient of the sample $^{11}$.Surber has derived the following relations for the dielectric parameters $D, \in{ }^{\prime}, \in{ }^{\prime \prime}$

$$
\begin{aligned}
& D=\tan \left[2 \tan ^{-1}\left(\frac{\alpha_{d} \lambda_{d}}{2 \pi}\right)\right] \\
& \in^{\prime}=\left(\frac{\lambda_{0}}{\lambda_{c}}\right)^{2}+\left(\frac{\lambda_{0}}{\lambda_{d}}\right)^{2}\left[1-\tan ^{2}\left(\frac{1}{2} \tan ^{-1} D\right)\right] \\
& \in{ }^{\prime \prime}=\frac{1}{\pi}\left(\frac{\lambda_{0}}{\lambda_{d}}\right)^{2} \alpha_{d} \lambda_{d}
\end{aligned}
$$

Where $\mathrm{D}$ is the dissipation factor, $\alpha_{d}$ is the attenuation constant due to dielectric, $\lambda_{d}$ is the wave length of the e.m. wave in the wave guide filled with the dielectric $\lambda_{0}$ is the free space wavelength, $\lambda_{c}=2 a$ is the cut off wavelength for the wave guide. $\alpha_{d} \lambda_{d}$ is the attenuation per wavelength. Having determined $\alpha_{d} \lambda_{d}, \lambda_{0} \lambda_{c}$ and $\lambda_{d}$ the values of $\mathrm{D}, \in^{\prime}, \in^{\prime \prime}$ may be calculated by using the equations (1), (2) and (3) respectively.

The density of pure components and their mixtures were measured by using DMA 35 portable vibrating density meter, Anton Paar, Austria (Europe). The parts of enhanced ULA adapter : ULA-49 EAY water jacket, sample chamber, tube end cap ULA-34, ULA-31 EY, ULA-31 EYZ, clamping collar ULA-OZE of Brook field engineering laboratories USA and low temperature circulating water bath, Nivtech Instruments \& Engineers, Thane, India at $30^{\circ} \mathrm{C}$. Specification of density meter, Anton Paar Austria is accuracy $0.001 \mathrm{~g} / \mathrm{cm}^{3}$ measuring range density 0 to $3 \mathrm{~g} / \mathrm{cm}^{3}$. Temperature 0 to $40^{\circ} \mathrm{C}$.

Viscosity of pure components and their mixtures were measured by using viscometer Brook field DVII + Pro model LVDV - II + P Brook field engineering laboratories, INC, USA, calibration of this instrument will be accurate to within $\pm 1 \%$ of its full scale range.

The refractive index of the pure components and their mixtures were measured by using Abbe's refractometer (with Glass scale) Mittal Enterprises, New Delhi, India, having an accuracy 0.001 by reading and 0.0001 by estimation. Measuring range extends from 1.300 to 1.700 with the help of sodium D line surface tension of pure components and their mixtures were measured by using Stalagmometer at room temperature $30^{\circ} \mathrm{C}$.

Acetonitrile (AR grade) purity (GC) $\leq 10$. Identity IR supplied by Merck KGaA, Darn Stadt, Germany and methanol (AR Grade) methanol laboratory Reagent >=99.6\% (GC) Supplied by sigma-Aldrich Steinheim Germany were used without further purification.

The solutions were prepared by mixing acetonitrile + methanol in volume. These binary liquid mixtures according to their proportions were mixed well and kept for 6 hours in a well stoppered bottle to ensure good thermal equilibrium. Microwave input and output power measured by Pm-437 (Attest) power meter, Chennai, India. Rectangular wave guide working $\Gamma_{E 10}$ mode $10 \mathrm{~dB}$, VidyutyantraUdyog, India.

Low temperature water circulating both was used for maintaining temperature of pure components and their binary liquid mixtures for measurement of viscosity, refractive index, density and plunger reading using Xband microwave bench.

\section{Results and Discussion}

Dissipation factor (D), dielectric constant $\left(\in^{\prime}\right)$, dielectric loss $\left(\in^{\prime \prime}\right)$, loss tangent ( $\tan \delta$ ), molar polarization $\left(p_{12}\right)$, a.c. conductivity $(\sigma p)$ apparent polarization $\left(\mathrm{P}_{2}\right)$ and activation energy $\left(E_{a}\right)$ for the viscous flow with increasing mole fraction $\left(\mathrm{X}_{\mathrm{A}}\right)$ of Acetonitrile for the binary mixture. Acetonitrile + Methanol 
are listed in Table 1.The values of viscosity $(\eta)$, refractive index $\left(n_{D}\right)$, density $(\rho)$, surface tension $(\mathrm{T})$, square of refractive index $\left(n_{D}^{2}\right)$, mole fraction of solute and solvent are listed in Table 2 .

The variation of the dielectric constant $\left(\in^{\prime}\right)$ with molar concentration of acetonitrile in the mixture is represented in figure 2.There is an increasing trend according to P. Job if dielectric constant $\left(\in^{\prime}\right)$ is plotted against the mole fraction for one of the components of a mixture the nature of the graph gives information regarding the occurrence of complexion. If the relationship observed is linear, then there is no occurrence of complexion.

On the other hand, if the two species are mixed and if complex form between the two species the value by additive property will pass through a maxima or minima the complex is at its greatest concentration at a point where the species are combined in the ratio in which they occurs in the complex. The curve of dielectric constant against mole fraction therefore shows a change in slope at the mole fraction corresponding to the complex. If the change in slope occurs at a mole fraction of 0.5 then it indicates a complex of the $1: 1$ type and if the change in slope occurs at a mole fraction of 0.7 then it indicates a complex of the 2:1 type.

In figure 2, the graph shows deviation from linearity, indicating complex formation in the mixture as suggested by P. Job. The deviation is maximum at about 0.534907 mole fraction of acetonitrile.

Hence, complex is at its greatest concentration at point $\mathrm{X}_{\mathrm{A}}=0.534907$ indicating the formation of $1: 1$ complex in the binary. This curve suggest that there is occurrence of intermolecular interaction between solute and solvent ${ }^{11}$ same behavior obtained ${ }^{12,}{ }^{13}$.It is observed that the dielectric constant $\left(\in \in^{\prime}\right)$ increases with increasing mole fraction of acetonitrile in the binary liquid mixtures of Acetonitrile+methanol.Similar values of $\in^{\prime}$ for pure methanol is obtained ${ }^{10}$.

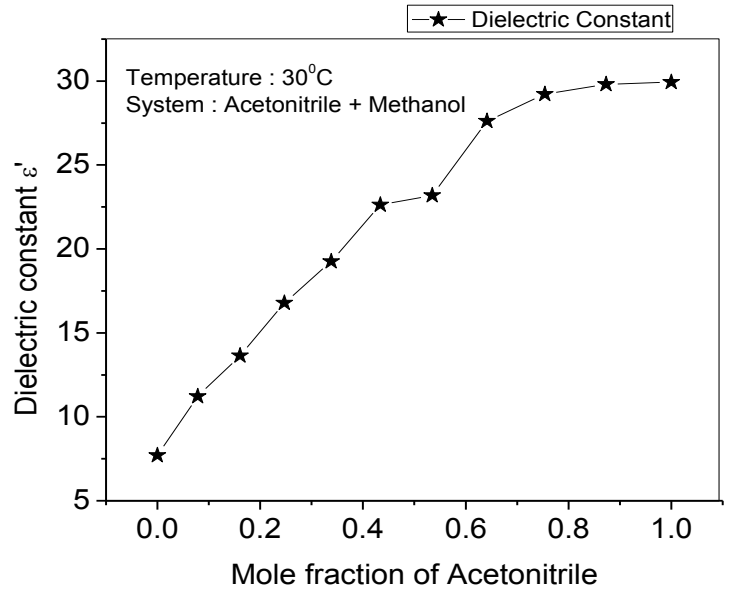

Figure 2: Variation of $\in{ }^{\prime}$ versus mole fraction of Acetonitrile in the mixture Loss tangent $(\tan \delta)$ :

The graph of loss tangent ( $\tan \delta$ ) versus mole fraction $\left(\mathrm{X}_{\mathrm{A}}\right)$ of acetonitrile is depicted in figure 3. The graph indicates that loss tangent $(\tan \delta)$ decreases with increasing mole fraction $\left(\mathrm{X}_{\mathrm{A}}\right)$ of acetonitrile in the mixture.

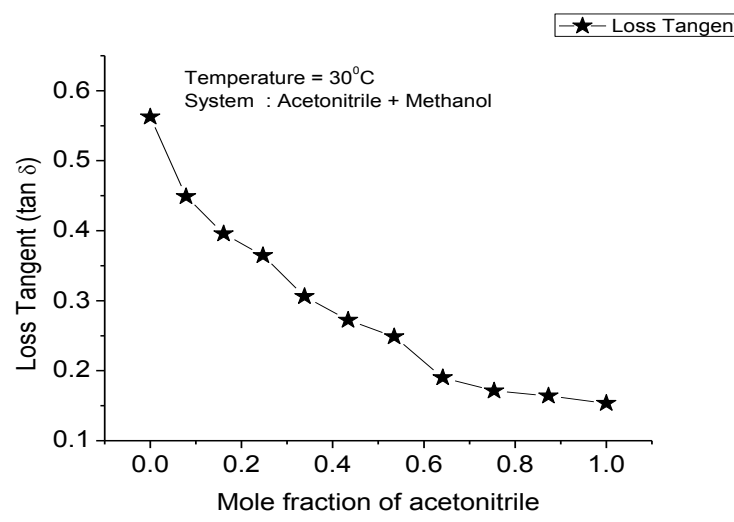

Figure3. Variation of Loss Tangent $(\tan \delta$ ) versus Mole fraction of acetonitrile in the mixture Molar and Apparent polarization 
The values of molar polarization $\mathrm{P}_{12}$ of the mixtures were obtained by the formula.

$$
P_{12}=\frac{\left(\in \epsilon^{\prime}-1\right)}{\left(\in^{\prime}+2\right)} \frac{\left(X_{A} M_{1}+X_{B} M_{2}\right)}{\rho}
$$

Where $M_{1}$ and $M_{2}$ are the molecular weights $X_{A}$ and $X_{B}$ are the mole fraction of solute and solvent respectively and $\rho$ is the density of the mixture. $\left(\in^{\prime}\right)$ dielectric constant of the binary liquid mixtures.

Apparent polarization $\mathrm{P}_{2}$ of the mixtures were obtained by the formula

$$
P_{2}=\frac{P_{12}-X_{A} P_{1}}{X_{B}}
$$

Where $\mathrm{P}_{12}$ is the molar polarization of mixtures, $\mathrm{P}_{1}$ is molar polarization of pure solvent, $X_{A}$ and $X_{B}$ are mole fraction of solute and solvent respectively.

Solvent - Solvent interaction between types of polar protic - polar aprotic solvent is due to hydrogen bonding formation as a result of amphiprotic hydrogen bond acceptor-donor (HBA-D). These solvent-solvent interaction property is depends mainly on different physical properties of solvent such as dielectric constant ( $\left.\in^{\prime}\right)$, dipole moment, molar polarization $\left(\mathrm{P}_{12}\right)$, donor number, chemical structure solvatochromic quantitative values of Kamlet Taft hydrogen bond acidity, basicity anddipolarity - Polarizability ${ }^{4}$.

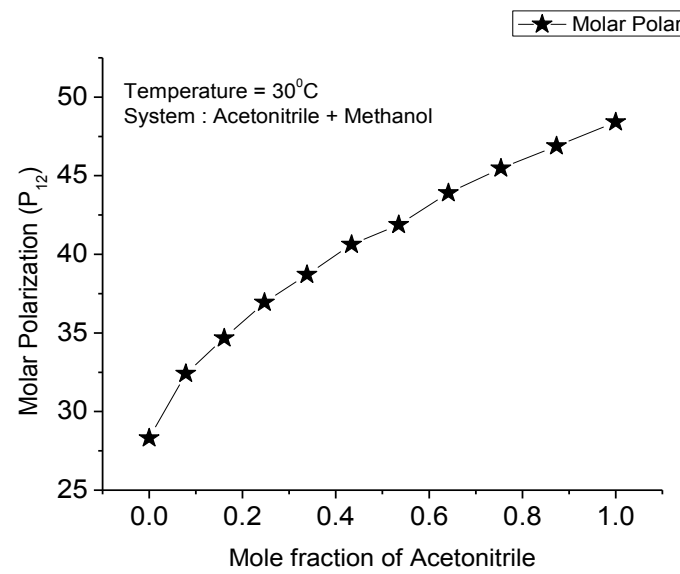

Figure 4. Variation of Molar Polarization $\left(P_{12}\right)$ versus Mole fraction of acetonitrile in the mixture

In present investigation as concentration of acetonitrile in the system increases dielectric constant $\left(\in^{\prime}\right)$ of the acetonitrile + Methanol system increases with molar polarization increases it is due to the formation of KamletTalf hydrogen bonding between Acetonitrile and methanol binary system.

As the dielectric constant $\left(\in^{\prime}\right)$ increases molar polarization also increases it indicate that the solventsolvent interaction is increases. This is due to formation of hydrogen bonding and this occurred due to the increasing the concentration of more polar solvent.

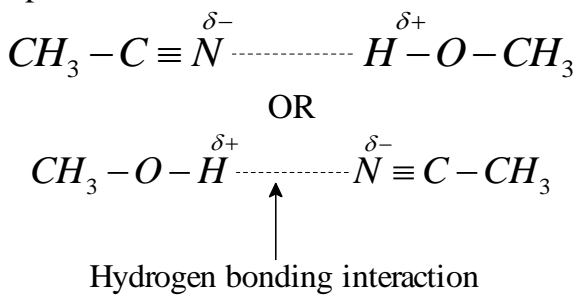

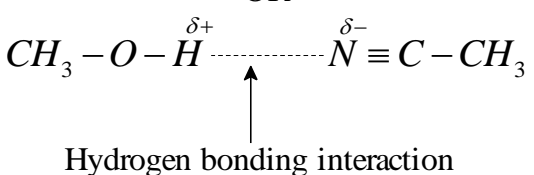

The values of molar polarization $\left(P_{12}\right)$ are plotted against the mole fraction $\left(\mathrm{X}_{\mathrm{A}}\right)$ of acetonitrile shown in figure (4). In our present investigation of acetonitrile + Methanol mixture the amount of complex present is responsible for the nature of polarization curve. Thus this result regarding the formation of complex supported by our earlier conclusion made for the $\epsilon^{\prime}$ versus mole fraction of $X_{A}$ plot. Deviation is maximum at $\mathrm{X}_{\mathrm{A}}=0.534907$ Mole fraction of acetonitrile in the binary liquid mixture of acetonitrile + methanol. Hence complex is at greatest concentration at $\mathrm{X}_{\mathrm{A}}=0.534907$ indicating the formation of $1: 1$ complex in the binary liquid mixture. Similar result have been obtained ${ }^{14}$. 
Apparent polarization $\left(\mathbf{P}_{2}\right)$ :

The graph of apparent polarization $\left(\mathrm{P}_{2}\right)$ versus mole fraction $\left(\mathrm{X}_{\mathrm{A}}\right)$ of acetonitrile is depicted in figure 5 . The graph indicates that apparent polarization decreases with increasing mole fraction $\left(\mathrm{X}_{\mathrm{A}}\right)$ of acetonitrile in the mixture.

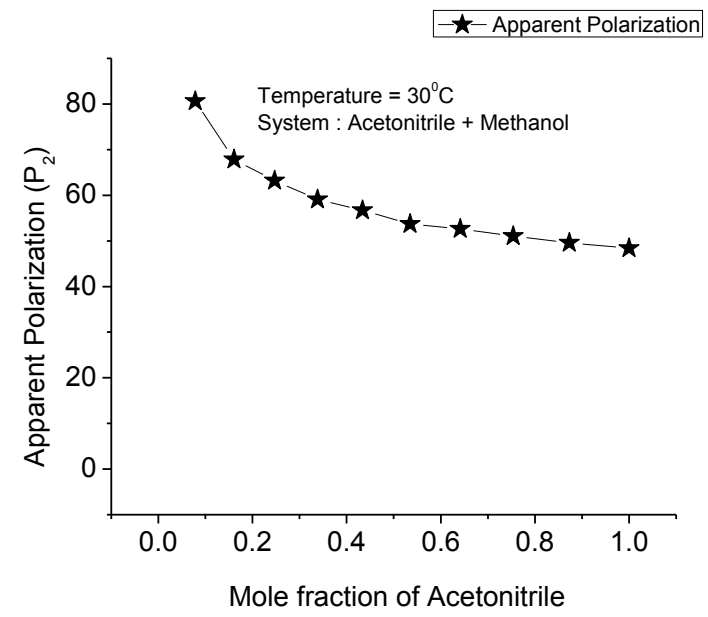

Figure5. Variation of apparent polarization $\left(\mathbf{P}_{2}\right)$ versus Mole fraction of acetonitrile in the mixture

Table No.1. Mole Fraction of Solute $\left(\mathbf{X}_{\mathrm{A}}\right)$, Dissipation factor $(\mathbf{D})$, Dielectric Constant $\left(\in \in^{\prime}\right)$, Dielectric loss ( $\in ")$, Loss Tangent $(\tan \delta)$, Molar Polarization $\left(\mathbf{P}_{12}\right)$, a.c. Conductivity $\left(\sigma_{p}\right)$, apparent Polarization $\left(\mathbf{P}_{2}\right)$ and Activation Energy (Ea) of Binary Liquid Mixtures at $30^{\circ} \mathrm{C}$

\begin{tabular}{|c|c|c|c|c|c|c|c|c|c|}
\hline $\begin{array}{c}\text { Sr. } \\
\text { no. }\end{array}$ & $\mathbf{X}_{\mathbf{A}}$ & $\mathbf{D}$ & $\epsilon^{\prime}$ & $\epsilon^{\prime \prime}$ & $\tan \delta$ & $\mathbf{P}_{\mathbf{1 2}}$ & $\sigma_{p}$ & $\mathbf{P}_{\mathbf{2}}$ & $\mathbf{E a}$ \\
\hline 1 & 0 & 0.591284 & 7.705522 & 4.335968 & 0.562709 & 28.307365 & 2.593123 & 0 & 5.690382 \\
\hline 2 & 0.078505 & 0.464315 & 11.225008 & 5.039032 & 0.448911 & 32.414324 & 3.01359 & 80.621982 & 5.65193 \\
\hline 3 & 0.160852 & 0.406935 & 13.645505 & 5.401292 & 0.395829 & 34.666704 & 3.230239 & 67.842708 & 5.610854 \\
\hline 4 & 0.247329 & 0.372963 & 16.788696 & 6.122681 & 0.364691 & 36.93315 & 3.661666 & 63.183118 & 5.589215 \\
\hline 5 & 0.338256 & 0.312257 & 19.248264 & 5.894131 & 0.306216 & 38.70742 & 3.524982 & 59.053471 & 5.574344 \\
\hline 6 & 0.433985 & 0.27672 & 22.622302 & 6.156992 & 0.272165 & 40.625408 & 3.682185 & 56.690934 & 5.566769 \\
\hline 7 & 0.534907 & 0.252578 & 23.193409 & 5.764088 & 0.248523 & 41.886387 & 3.447209 & 53.693127 & 5.559097 \\
\hline 8 & 0.641456 & 0.192714 & 27.624985 & 5.251968 & 0.190117 & 43.902986 & 3.140936 & 52.620211 & 5.551327 \\
\hline 9 & 0.754116 & 0.173523 & 29.222987 & 5.006236 & 0.171312 & 45.476408 & 2.993976 & 51.074476 & 5.543454 \\
\hline 10 & 0.873428 & 0.166031 & 29.80699 & 4.887068 & 0.163957 & 46.887221 & 2.922708 & 49.579703 & 5.535478 \\
\hline 11 & 1 & 0.155435 & 29.93533 & 4.595124 & 0.153502 & 48.416779 & 2.748111 & 48.416779 & 5.527394 \\
\hline
\end{tabular}

a.c. Conductivity $\left(\sigma_{p}\right)$

a.c. conductivity of the mixture where obtained by using the formula

$$
\begin{aligned}
& \sigma_{p}=\omega \in_{0} \in " \\
& \sigma_{p}=2 \pi f \in_{0} \in "
\end{aligned}
$$

Where $\omega=2 \pi f$

$\mathrm{f}=10.75 \mathrm{GHz}$

$\in_{0}=8.854187816 \times 10^{-12} C^{2} / \mathrm{Jm}$

$\in "=$ Dielectric loss of binary liquid mixture

In our work, it is found that a.c. conductivity depends on dielectric loss $(\in ")$ dielectric loss decreases a.c. conductivity decreases and dielectric loss increases a.c. conductivity increases. 


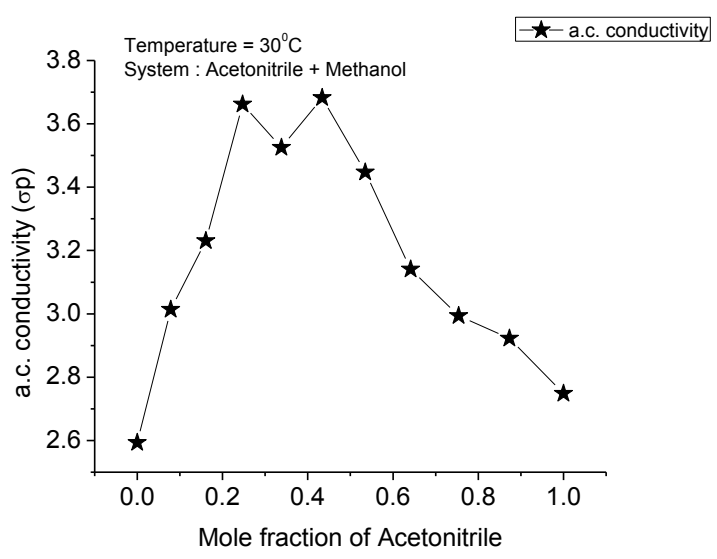

Figure6. Variation of a.c. conductivity $(\square$ p)

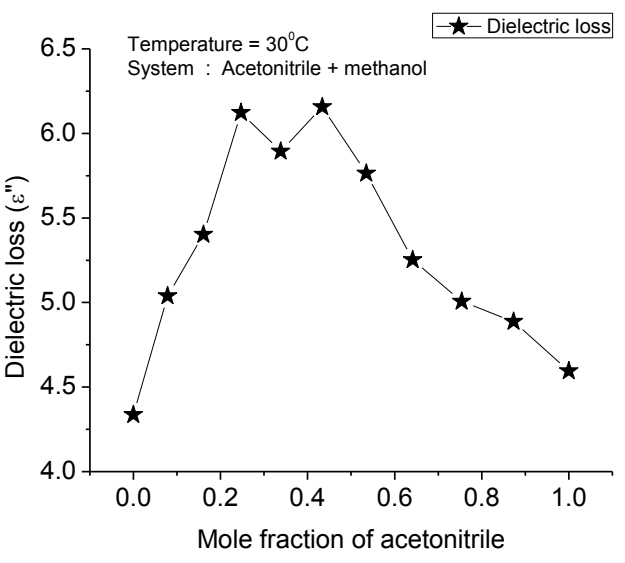

Figure7. Variation of Dielectric loss $(\in ")$ versus Mole fraction of acetonitrile in the mixture

Dielectric loss is proportional to the a.c. conductivity. Thus a.c. conductivity depends upon the dielectric $\operatorname{loss}^{15}$.a.c conductivity and dielectricloss is presented in figure (6) and (7) respectively.

Table: 2. Mole fraction of solute $\left(X_{A}\right)$, Mole Fraction of Solvent $\left(X_{B}\right)$, Density ( $\left.\square \square\right)$, Viscosity $(\square)$, Refractive Index $\left(\mathbf{n}_{\mathrm{D}}\right)$, Square of Refractive Index $\left(\left(\mathrm{n}_{\mathrm{D}}\right)^{2}\right)$ and Surface Tension (T) of Binary Liquid Mixtures at $30^{\circ} \mathrm{C}$

\begin{tabular}{|c|c|c|c|c|c|c|c|}
\hline Sr.no. & $\mathbf{X}_{\mathbf{A}}$ & $\mathbf{X}_{\mathbf{B}}$ & $\begin{array}{c}\square \\
\mathbf{g m} / \mathbf{c m}^{\mathbf{3}}\end{array}$ & $\begin{array}{c}\square \\
\mathbf{~ P}\end{array}$ & $\mathbf{n}_{\mathbf{D}}$ & $\left.\mathbf{( n}_{\mathbf{D}}\right)^{\mathbf{2}}$ & $\begin{array}{c}\mathbf{T} \\
\mathbf{d y n e} / \mathbf{c m}\end{array}$ \\
\hline 1 & 0 & 1 & 0.782 & 0.97 & 1.331 & 1.771561 & 18.824231 \\
\hline 2 & 0.078505 & 0.921495 & 0.7811 & 0.91 & 1.332 & 1.774224 & 20.306787 \\
\hline 3 & 0.160852 & 0.839148 & 0.7808 & 0.85 & 1.333 & 1.776889 & 21.034937 \\
\hline 4 & 0.247329 & 0.752671 & 0.7797 & 0.82 & 1.334 & 1.779556 & 21.678919 \\
\hline 5 & 0.338256 & 0.661744 & 0.7785 & 0.8 & 1.335 & 1.782225 & 22.692413 \\
\hline 6 & 0.433985 & 0.566015 & 0.7771 & 0.79 & 1.336 & 1.784896 & 23.59542 \\
\hline 7 & 0.534907 & 0.465093 & 0.7752 & 0.78 & 1.337 & 1.787569 & 24.425945 \\
\hline 8 & 0.641456 & 0.358544 & 0.7742 & 0.77 & 1.338 & 1.790244 & 25.356261 \\
\hline 9 & 0.754116 & 0.245884 & 0.7719 & 0.76 & 1.339 & 1.792921 & 25.621151 \\
\hline 10 & 0.873428 & 0.126572 & 0.7709 & 0.75 & 1.34 & 1.7956 & 26.57846 \\
\hline 11 & 1 & 0 & 0.7682 & 0.74 & 1.341 & 1.798281 & 25.982485 \\
\hline
\end{tabular}

\section{Refractive index $\left(\eta_{D}\right)$}

In general refractive index of compounds increases with increasing atomic number of the constituent atoms ${ }^{16}$ and furthermore the refractive index of a compound is a physical properly that can be potentially be used It is like a melting point, to establish its density very easily ${ }^{16}$.

The structural property of liquid and liquid mixtures can be integrated through the refractive indices employing mole fraction. In general refractive increases with molecular weight for symmetric and asymmetric molecules $^{17}$

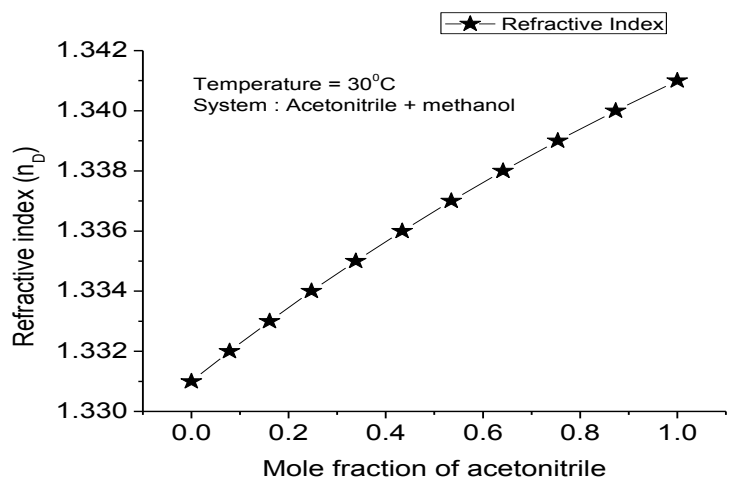

Figure8. Variation of Refractive index $\left(\mathrm{n}_{\mathrm{D}}\right)$ versus Mole fraction of acetonitrile in the mixture 
In our present work it is clear from figure (8) that the refractive index values are increasing with increasing concentration of acetonitrile in the binary liquid mixture of acetonitrile + methanol same behavior is obtained $^{19}$.Similar value of refractive index for acetonitrile was obtained ${ }^{5}$.

Viscosity $(\eta)$

In figure (9) the graph shows the viscosity decreased with increasing mole fraction of acetonitrile in the system acetonitrile + methanol at $30^{\circ} \mathrm{C}$ temperature.

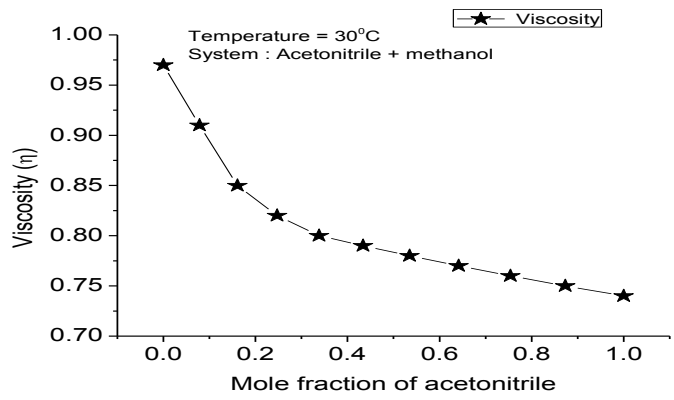

Figure9. Variation of Viscosity $(\eta)$ versus Mole fraction of acetonitrile in the mixture

Kenneth Hickey et.al. ${ }^{18}$ reported similar result. Patric NgoyTshibangu et.al. ${ }^{20}$ reported ionic liquid viscosity is ordinarily influenced by other interactions such as hydrogen bonding and the symmetry of the ions. Similar decreasing trend is obtained ${ }^{21,22}$ weak types of dipole induced dipole of interactions are not sufficient to product bulky or less mobile entities in system and hence decreased trend of viscosity is observed in the present binary liquid mixtures of acetonitrile + methanol.

Density $(\rho)$

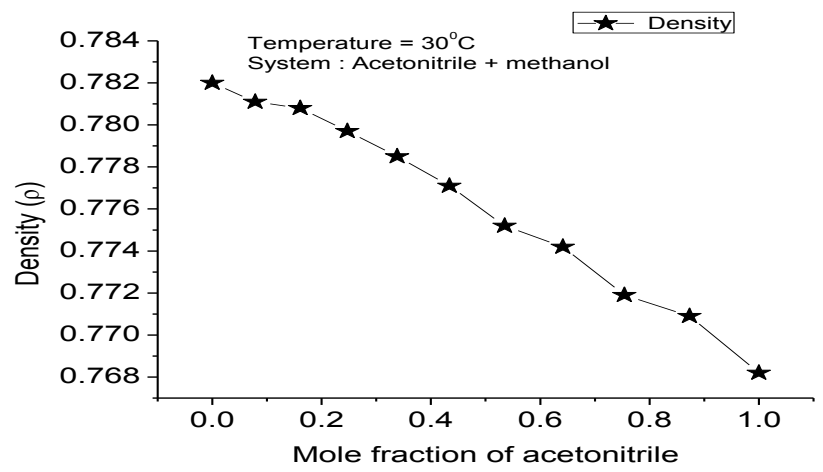

Figure10. Variation of Density $(\rho)$ versus Mole fraction of acetonitrile in the mixture

Figure (10) shows the density measurement curve obtained is linear. Density value decreased with increases mole fraction of acetonitrile in the binary liquid mixture of acetonitrile + methanol. Similar decreased trends of density is obtained ${ }^{22,23,24}$. Similar value of density of pure acetonitrile is obtained ${ }^{6}$.

Surface Tension

The values of mole fraction of acetonitrile versus surface tension of binary liquid mixture of acetonitrile and methanol are presented in figure (11). It is in increasing trend.

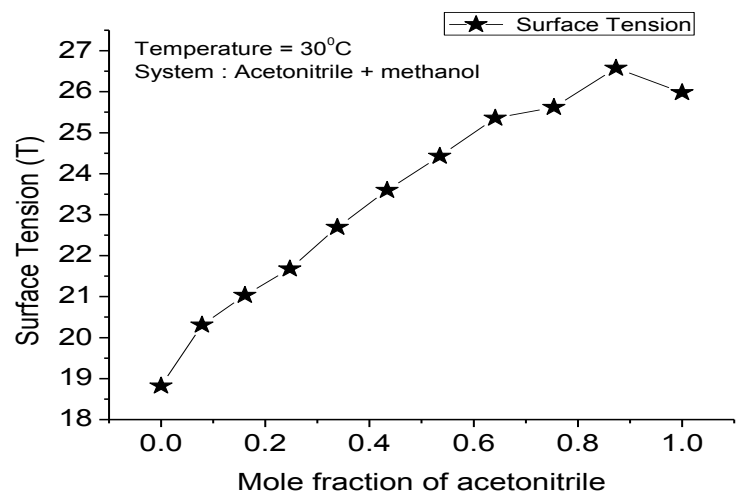

Figure11. Variation of Surface Tension (T) versus Mole fraction of acetonitrile in the mixture 
As it can be seen from figure (11), surface tension (T) is positive for the binary mixture of (acetonitrile +methanol) the strong interaction between acetonitrile + methanol give rise to an increase in surface tension.This is in turn responsible for the positive surface tension (T) for the (Acetonitrile + methanol) system. ${ }^{24}$.

\section{Activation Energy (Ea):}

In chemistry activation energy is term introducedin1889 by the Swedish scientist Arrhenius that is defined as the minimum energy that must be in put to a chemical system, containing potential reactants, in order for a chemical reaction to occur, Activation energy may also be defined as minimum energy required to start a chemical reaction. The activation energy of a reaction is usually denoted by Ea. Activation energy can be thought of as the height of the potential barrier9 sometimes called the energy barrier) separating two minima of potential energy of the reactants and products of a reaction). For a chemical reaction to proceed at a reasonable rate, there should exit an appreciable number of molecules with energy equal to or greater than the activation energy. The graph of Activation energy $(\mathrm{Ea})$ versus mole fraction $\left(\mathrm{X}_{\mathrm{A}}\right)$ of Acetonitrile is presented in figure (12). We observe that activation energy decreases with increasing mole fraction of Acetonitrile in the mixture. Same nature of graph is obtained. ${ }^{25}$

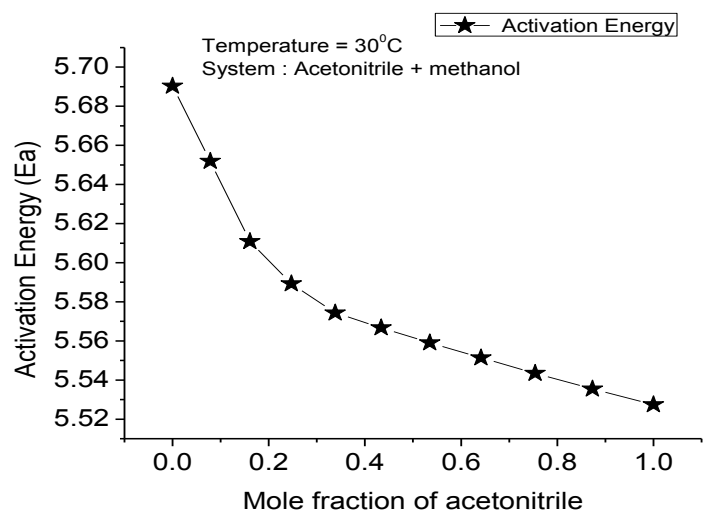

\section{Figure12. Variation of Activation Energy (Ea) versus Mole fraction of acetonitrile in the mixture}

\section{Conclusions}

The values of dielectric constant $\left(\in^{\prime}\right)$, Dielectric loss $\left(\in \in^{\prime}\right)$, loss tangent ( $\tan \delta$ ), molar polarization $\left(\mathrm{P}_{12}\right)$, Apparent polarization $\left(\mathrm{P}_{2}\right)$, activation energy $\left(\mathrm{E}_{\mathrm{a}}\right)$ andviscosity

$(\eta)$, refractive index, surface tension and density are all positive values indicating strong interactions between acetonitrile + methanol molecules.

There is intermolecular interaction among the components of the binary mixtures leading to hydrogen bond formation of the type 1:1 complex formation between acetonitrile + methanol mixtures.

\section{References}

[1]. S.V. Kumara Sastry, S. SreehariSastry and VRK Murthy, Excess dielectric and thermodynamical studies on hydrogen bonded binary mixtures of propan-1-ol with methyl and ethyl benzoates, Chem. Sci. Trans. 2012, 1 (3), 638-652.

[2]. RekhaPande\& G.M. Kalamse, Asian J. Chemistry Vol. 17, No. 1 (2005), 283-288.

[3]. Ch. V.V. Ramana, A.B.V. Kirankumar, M. Ashok kumar and M.K. Moodley Dielectric and excess dielectric constant of acetonitrile + Butyl amine + Ethylamine and methylamine at 303, 313, and $323 \mathrm{~K}$ Journal of chemistry volume 2013 (2013), Article ID 687106

[4]. Farid I. EI. Dossoki refractive index and density measurements for selected binary protic - protic, aprotic - aprotic, and aproticprotic systems at temperatures from $298.15 \mathrm{~K}$ to $308.15 \mathrm{~K}$. J. of the Chinese chemical society 2007, 54, 1129-1137.

[5]. Abeer K. Shams Densities, refractive indices and excess properties of binary mixtures of acetonitrile with Benzene, Toluene $\mathrm{n}$ xylene and mesitylene at temperatures from (298.15 to 313.15 K), Journal of Al. Nahrain University Vol. 14 (2), June, 2011, Pp. $75-85$.

[6]. R.K. Sukla, G.K. Gupta, S.K. Puranik, A.K. Sharma and Balwant Singh, surface tension of Binary liquid mixtures from various liquid state models at 293.15, 298.15 and 313.15 K, Int. J. of Latest Research in Science and Technology vol. I. Issue 3 Page NO. 276-281 Sept-Oct (2012)

[7]. Ch. V.V. Ramana G. Ramachandra Reddy and M. Mohan Reddy, Dielectric and excess dielectric constant of acetonitrile + hexane + heptanes and + cyclohexane at 303-313 and 323 K, Physical chemistry, Vol. 4, No. 2, 2009.

[8]. W.H. Surber, 1, Appl. Phys. 19, 514 (1948)

[9]. P.J. Singh and K.S. Sharma Dielectric behaviour of ketone-amine binary mixtures at microwave frequencies pramana Journal of Physics Vol. 46, No.4, April 1996, Pp. 259-270.

[10]. Mohammed N. Afsar, Nattakarn, Suwanvisan, and Yong wang permittivity measurement of low and high loss liquids in the freq. range of 8 to $40 \mathrm{GHz}$ using wave guide transmission line technique microwave and optical technology letters. Vol. 48, NO. 2, 275281, Feb. 2006

[11]. P. Job Ann. Chem. 9, 113, (1928) 
[12]. R.S. Kawale, U.B. Tumbarphale, V.G. Kalamse and G.M. Kalamse, Dielectric behaviour of thiophenol in benzene, carbon tetrachloride, cyclohexane, N-hexane,Internation J. of Physics \& Mathematical Sciences ISSN : 2277-2111, 2013, Vol. 3 (2), April-June, pp. 99-103/ Kawle et.al.

[13]. B.S. Narwade, P.G. Gawali, RekhaPande and G.M. Kalamse, Dielectric studies of binary mixtures of n-propyl alcohol and ethylenediamine J. Chem. Soci. Vol. 117, No. 6, Nov. 2005, Pp. 673-676.

[14]. NarwadeBhanudasSheshrao,Thermodynamic properties and related studies of polar liquids at microwave frequencies, thesis SRTMU Nanded, Maharashtra India, 2006, P.No. 45-46.

[15]. H.C. Chaudhari and V.J. Shinde, Dielectric properties of soils at X-band microwave frequencies, Indian Journal of Pure and applied physics vol.50 January 2012, pp. 64-66.

[16]. Oscar cabeza, Sandra Garcia-Garabad, Luisa segade, montservat, Domingue-Perez Esther pilo and Luis M. Varela, 2011. Universidada da coruna, universidadesatiago de comnaspain Physical properties of Binary mixtures of ILS with water and ethanol A review (Roger and Seddon) ionic liquids. Theory properties, Newapproatues prof. Alexander Kokorin (Ed.) ISBN 978-303-349 In. Tech.

[17]. K. Narendra, P. Narayanamurthy and Ch. Shrinivasu 2011, Refractive indices of binary liquid mixture at different temperature Asian Journal of Applied Sciences 4, 535-541.

[18]. Kenneth Hickey and W. Earle Waghore, Viscosities and Volumes of dilute solutions of formamide in water + acetonitrile and for formamide and $\mathrm{N}, \mathrm{N}$-dimehtylformamide in methanol + acetonitrile mixed solvents : Viscosity B-coefficients, activation free energies for viscous flow and partial molar volumes, J. Chem. Eng. Data 2001, 46, 851-857.

[19]. Jeevanandham P, Kumar S. Periyasamy, P. and Sedhumatharen K. Dielectric properties of binary liquid mixtures of aniline, ochloroaniline and o-anisidine with carbon tetrachloride at $303.15 \mathrm{~K}$, Int. J. of Recent Scientific research Vo. 3 Issue 10 pp. 878 883, October 2012.

[20]. Patrick NgoyTshibangu, Silindile, Nomatheruba Ezekiel Dixon Dikio. Density, Viscosity and conductivity, study of 1-Butyle-3methylimidazolium Bronide Int. J. Electro Chem. Sci. 6 (2011) 2201-2213.

[21]. Sujata S. Patil and Sunil R. Mirgane, Thermodynamics of binary liquid mixtures of acrylates with dodecane-1-ol Pelagia Research Library. Advances in applied science research 2013, (4), 242-250, ISSN : 0976-8610, Coden (USA) AASRC

[22]. Chandra mohanSaxena, ArchanaSaxena, Ashok Kumar Srivastava and Noveen Kumar Shukla, Densities, voscosites and ultrasonic studies of binary liquid mixture of thylamine and Benzyl alcohl at different temperatures, American chemical science journal 3 (4), 468-478, 2013, Science Dome in international.

[23]. George Rizoulis, Excess properties of the binary liquid systems dimethyl sulfoxide + isopropanol and propylene carbonate + isopropanol, Can J. Chem. 67 (1989), 1105.

[24]. SeyedHesammirfakhar, DavoodRezaei- Teimourbagloo, Density and surface tension of binary mixture of acetonitrile, 2-Alkanol at 298.15K, Journal of science and Today's world, 2013 volume 2, issue 4, pages 344-357.

[25]. Kh. Abdul Maleque Effects of Binary chemical reaction and Activation energy on MHD Boundary layer Heat and mass Transfer flow with viscous Dissipation and Heat Generation /Absorption., Hindawi publishing corporation ISRN Thermodynamics Volume 2013, Article ID 284637, 9 pages http://dx.doi.org/10.1155/2013/284637. 